\title{
PREDICCIÓN NUTRICIONAL PARA PASTOS TROPICALES POR ESPECTROSCOPÍA DE REFLECTANCIA EN EL INFRARROJO CERCANO $^{1}$
}

\author{
Luis Alberto Sandoval-Mejía ${ }^{2}$,Francisco Javier Bueso-Uclés², Miguel Vélez-Nauer ${ }^{2}$
}

\begin{abstract}
RESUMEN
Predicción nutricional para pastos tropicales por espectroscopía de reflectancia en el infrarrojo cercano. Muestras de pastos tropicales $(n=155)$ fueron recolectadas de cuatro países centroamericanos. Las muestras fueron secadas a $60{ }^{\circ} \mathrm{C}$ por 48 horas, molidas y escaneadas por espectroscopía de reflectancia en el infrarrojo cercano (NIRS) y analizadas para proteína cruda (PC) fibra neutro detergente (FND) y fibra ácido detergente (FAD) por métodos químicos de referencia. El estudio fue realizado de enero a septiembre del 2005. Fueron utilizadas ciento treinta y una muestras para la calibración y validación cruzada usando el programa WINISI, mientras que las restantes se utilizaron para la validación externa contra métodos químicos de referencia. Las muestras utilizadas para la calibración tuvieron entre 1,9 y $22 \%$ de proteína cruda con una media de $9,85 \%$. Amplios rangos de contenido para FND y FAD estuvieron igualmente presentes en las 131 muestras usadas para la calibración. La espectroscopía de reflectancia en el infrarrojo cercano predijo con exactitud el contenido de PC, FND y FAD de las muestras utilizadas para la validación. Altos coeficientes de determinación $\left(\mathrm{R}^{2}\right)$ y bajos errores estándar de validación (EEV) fueron obtenidos para PC $(0,98$; $0,25)$, FAD $(0,90 ; 0,96)$ y FND $(0,92 ; 0,60)$. Los coeficientes de variación de las predicciones de NIRS para PC, FAD y FND $(4,5 ; 1,5$ y $1,5 \%$, respectivamente), indicaron buena precisión durante las predicciones. Un grupo mayor de muestras de pastos es necesario para validar las ecuaciones NIRS en la región centroamericana.
\end{abstract}

Palabras clave: Proteína cruda, fibra neutro detergente, fibra ácido detergente, regresión por cuadrados mínimos parciales modificados, NIRS.

\begin{abstract}
Tropical forage grasses nutrient prediction by nearinfrared reflectance spectroscopy. Tropical forage grass samples $(n=155)$ were collected from four Central American countries. Samples were dried at $60{ }^{\circ} \mathrm{C}$ for $48 \mathrm{~h}$, milled and scanned trough a near-infrared reflectance spectrophotometer (NIRS) and analyzed for crude protein (CP), acid detergent fiber (ADF) and neutral detergent fiber (NDF) by reference chemistry methods. The study was performed from January to September, 2005. A set of 131 samples were used for NIRS calibration an internal cross validation performed by WINISI software, while the remaining 24 samples were used for validation against reference methods. The $\mathrm{CP}$ of samples used for NIRS equation development covered a wide range from 1.9 to $22 \%$ with a mean of $9.85 \%$. Wide ranges for $\mathrm{ADF}$ and NDF content were also observed in the 131-sample calibration set. Near-infrared reflectance spectroscopy accurately predicted $\mathrm{CP}, \mathrm{ADF}$ and NDF of the tropical forages used for the validation. Coefficients of determination $\left(\mathrm{R}^{2}\right)$ and standard error of validation (SEV) for CP, ADF and NDF were as follows (0.98 and 0.25, 0.90 and $0.96,0.92$ and 0.60$)$. Percent coefficient of variation was low for NIRS predicted CP, ADF and NDF (4.5, 1.5 and $1.5 \%$ respectively), indicating good precision. A larger set of forage samples is needed to validate NIRS equations in the Central American region.
\end{abstract}

Key words: Crude protein, neutral detergent fiber and acid detergent fiber, modified least squares regression, NIRS.

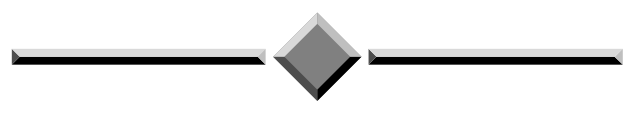

\footnotetext{
Recibido: 14 de agosto, 2007. Aceptado: 26 de agosto, 2008. Proyecto de Tesis para optar al título de Ingeniero Agroindustrial en el grado académico de Licenciatura. Escuela Agrícola Panamericana/Zamorano.

2 Carrera de Agroindustria y Carrera de Ciencia y Producción Agropecuaria. Escuela Agrícola Panamericana/Zamorano. Apartado Postal 93, Tegucigalpa, Honduras. sandovalmejia@gmail.com; fbueso@zamorano.edu; mvelez@zamorano.edu
} 


\section{INTRODUCCIÓN}

Los pastos tropicales representan la principal fuente de alimento en ganaderías tropicales en Centro América. Desde principios de los 90's, los ganaderos Centroamericanos han estado renovando sus pasturas mediante la introducción de variedades tropicales, especialmente híbridos de Brachiarias de origen africano, obteniendo mayores rendimientos en suelos ácido y mejoras en la digestibilidad (Lascano 2002). El análisis de calidad de los pastos tropicales de la región Centroamericana todavía se realiza por medios químicos debido a la falta de curvas de calibración NIRS (Near-Infrared Reflectance Spectroscopy, por sus siglas en inglés) para la rápida determinación de Proteína Cruda (PC), Fibra Ácido Detergente (FAD) y Fibra Neutro Detergente (FND).

La exactitud y precisión de NIRS para la predicción de la composición nutricional (PC, FAD y FND) de pastos y forrajes ha sido ampliamente estudiada en América del Norte (Brooks et al. 1984, Norris et al. 1976), España (Garcia-Ciudad et al. 1993), las planicies semi-áridas de Australia (Woolnough y Foley 2002) y las sabanas tropicales de Colombia (Lascano 2002, Vásquez et al. 2004). La mayoría de las curvas de predicción NIRS para la composición nutricional de pastos tropicales disponibles en Latinoamérica, han sido desarrolladas por centros de investigación para el análisis específico de pastos como Brachiaria spp (Lascano 2002) о Panicum maximum (Vasquez et al. 2004).

Altos coeficientes de determinación $\left(\mathrm{R}^{2}>0,96\right)$ y bajos errores estándar de calibración $(\mathrm{EEC}<1)$ han sido obtenidos para PC (Norris et al. 1976; Brooks et al. 1984; Woolnough y Foley 2002). Curvas de predicción NIRS para FAD y FND usualmente tienen $\mathrm{R}^{2}$ entre 0,85 y 0,95 y EEC entre 0,1 y 2,64 (Brooks et al. 1984, Garcia-Ciudad et al. 1993, Norris et al. 1976, Woolnough y Foley 2002, Vasquez et al. 2004). Los altos $\mathrm{R}^{2}$ y bajos EEC son obtenidos mediante regresiones usando Cuadrados Mínimos Parciales Modificados (CMPM) y tratamientos matemáticos de los espectros de las muestras que incluyen primera y segunda derivada (Cozzolino 2002, Cozzolino et al. 2003, Woolnough y Foley 2002).

El objetivo de este estudio fue explorar la exactitud y precisión de NIRS para la predicción de PC,
FAD y FND en un amplio rango de pastos tropicales cultivados en la región centroamericana para ganado de carne y leche.

\section{MATERIALES Y MÉTODOS}

El estudio fue realizado de enero a septiembre de 2005 en el Laboratorio de Análisis de Alimentos de la Escuela Agrícola Panamericana, Zamorano. Ubicado en el Valle del Yeguare, departamento de Francisco Morazán, Honduras.

\section{Preparación de las muestras}

Ciento cincuenta y cinco muestras de pastos colectadas de enero a julio de 2005, fueron seleccionadas del total de muestras enviadas al Laboratorio de Análisis de Alimentos de Zamorano para análisis de rutina. Las muestras incluyeron 38 Panicum maximum cultivar tobiata, 32 híbrido Brachiaria cultivar mulato, 30 Digitaria decumbens cultivar transvala, 22 Panicum maximum cultivar tanzania, 11 Brachiaria brizantha cultivar toledo, nueve Pennisetum purpureum cultivar king grass, cinco Andropogon gayanus, cinco de $C y$ nodon nlemfuensis y dos Tripsacum laxum recolectadas en Honduras, Nicaragua, El Salvador y Guatemala. Las edades de corta fueron de los 14 a los 42 días.

Las muestras fueron homogenizadas, secadas a $60^{\circ} \mathrm{C}$ por 48 horas y molidas con un molino Thomas Wiley, modelo 4 (Arthur H. Thomas Co., Philadelphia, PA), y una malla de $1 \mathrm{~mm}$. Finalmente, las muestras fueron colectadas en bolsas plásticas de nylon cierra fácil, para ser almacenadas a temperatura ambiente hasta su análisis.

\section{Análisis por los métodos de referencia}

Las muestras molidas fueron secadas a $105^{\circ} \mathrm{C}$ en un horno de convección por 24 horas para determinar la humedad residual de acuerdo al método 930.15 de la AOAC (AOAC 2005). La PC, FAD y FND fueron determinadas por los métodos 984,13; 973,18 y 2002,04 de la AOAC respectivamente (AOAC 2005). Los análisis de referencia fueron realizados por duplicado y reportados como porcentaje en base seca. 


\section{Calibración del NIRS y análisis de regresión}

Las muestras molidas fueron colocadas en contenedores rectangulares de plástico con ventana de cuarzo para su análisis por duplicado en un espectrofotómetro de reflectancia en infrarrojo cercano (Modelo 5000, FOSSNIR System, Silver Spring, MD) entre los 1100 y $2498 \mathrm{~nm}$. Los espectros de las muestras fueron tomados en una habitación a $21^{\circ} \mathrm{C}$ y $30 \%$ de humedad relativa.

Ciento treinta y una muestras fueron utilizadas para la calibración y validación cruzada realizadas con el software WINISI ${ }^{\circledR}$ v 1.50. Las curvas de calibración para PC, FAD y FND fueron generadas usando regresión por Componentes Principales (CP), Cuadrados Mínimos Parciales (CMP) y Cuadrados Mínimos Parciales Modificados (CMPM) relacionando la información espectral y los análisis de referencia. Cuatro transformaciones matemáticas $(2,5,5 ; 2,10,10 ; 1,4,4$ y $1,5,5)$ recomendadas por Cozzolino et al. (2003) fueron evaluadas en combinación con cada método de regresión. La selección de las ecuaciones para PC, FAD y FND se basó en el coeficiente de determinación más alto $\left(\mathrm{R}^{2}\right)$ y el Error Estándar de Calibración (EEC) más bajo.

\section{Validación Externa de las Ecuaciones del NIRS}

Veinte y cuatro muestras no utilizadas durante la calibración fueron utilizadas para determinar la exactitud y precisión del NIRS para determinar PC, FND y FAD en comparación con los métodos químicos de referencia. Un análisis de regresión usando SAS ${ }^{\circledR}$ V8 (1995) fue realizado para establecer la exactitud de los valores de PC, FND y FAD obtenidos con las ecuaciones del NIRS con respecto a los métodos químicos de referencia. El coeficiente de determinación de la regresión $\left(\mathrm{R}^{2}\right)$ fue usado como parámetro de exactitud de las ecuaciones del NIRS. Error Estándar de Validación (EEV) y el porcentaje del coeficiente de variación $(\% \mathrm{CV})$ fueron parámetros usados para comparar la precisión de las ecuaciones del NIRS versus los análisis químicos de referencia.

\section{RESULTADOS Y DISCUSIÓN}

\section{Composición de las muestras}

Los resultados de los análisis de laboratorio son presentados en el Cuadro 1. Las muestras de PC utilizadas para el desarrollo de la ecuación se encontraban en el rango de 1,9 a $22 \%$ con una media de $9,85 \%$. El contenido de FND y FAD igualmente presentó un amplio rango de variación en el grupo de las 131 muestras utilizadas para la calibración.

Cuadro 1. Composición nutricional de los pastos obtenida por los métodos químicos de referencia. Escuela Agrícola Panamericana/Zamorano, Honduras. 2005.

\begin{tabular}{lcccc}
\hline \multicolumn{1}{c}{ Componente } & Media & Mínimo & Máximo & DE $^{\mathbf{1}}$ \\
\hline PC, $\%$ de MS $^{2}$ & 9,85 & 1,89 & 22,33 & 3,49 \\
FND, \% de MS & 59,83 & 41,52 & 73,27 & 5,55 \\
FAD, \% de MS & 37,69 & 26,15 & 52,39 & 4,67 \\
\hline
\end{tabular}

${ }^{1}$ DE - Desviación Estándar.

${ }^{2} \mathrm{MS}$ - Materia seca.

\section{Resultados de la Calibración del NIRS}

Una distribución uniforme del espectro NIRS en el rango usado para calibración de PC, FND y FAD fue obtenido de las muestras de pastos usadas para este estudio (Figura 1). La uniformidad de la distribución es importante para asegurar la exactitud al predecir el contenido nutricional en el rango de calibración.

Ecuaciones obtenidas por regresión con base en los CMPM tuvieron mejores $\mathrm{R}^{2}$, que regresiones por CMP y CP (Cuadro 2). La transformación matemática 1,5,5 de la información espectral del NIRS junto con la regresión por CMPM produjo la ecuación con el $\mathrm{R}^{2}$ más alto para la composición nutricional de pastos, similares resultados fueron obtenidos por Cozzolino et al. (2003). Esta ecuación fue seleccionada para realizar ambas validaciones, cruzada y externa, contra los métodos químicos de referencia. 


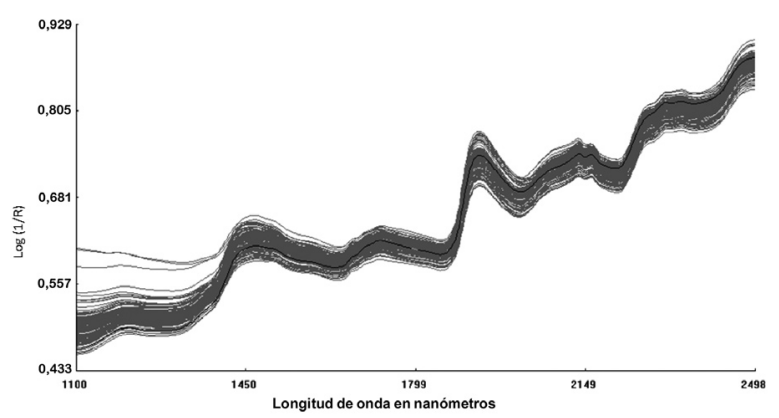

Figura 1. Espectro NIRS en 131 muestras de pastos tropicales. Universidad Zamorano, Honduras. 2005.

Cuadro 2. Coeficientes de determinacion $\left(\mathrm{R}^{2}\right)$ de las regresiones NIRS para los componentes nutricionales de 131 muestras de pastos tropicales. Universidad Zamorano, Honduras. 2005.

\begin{tabular}{ccccc}
\hline $\begin{array}{c}\text { Método de } \\
\text { Regresión }^{1}\end{array}$ & Transformación $^{2}$ & PC & FND & FAD \\
\hline \multirow{2}{*}{ RCP } & $2,5,5$ & 0,87 & 0,87 & 0,81 \\
& $2,10,10$ & 0,90 & 0,90 & 0,86 \\
& $1,4,4$ & 0,91 & 0,88 & 0,84 \\
& $1,5,5$ & 0,91 & 0,88 & 0,84 \\
CMP & $2,5,5$ & 0,93 & 0,93 & 0,89 \\
& $2,10,10$ & 0,91 & 0,93 & 0,89 \\
& $1,4,4$ & 0,92 & 0,95 & 0,88 \\
& $1,5,5$ & 0,92 & 0,95 & 0,88 \\
CMPM & $2,5,5$ & 0,93 & 0,94 & 0,89 \\
& $2,10,10$ & 0,93 & 0,95 & 0,88 \\
& $1,4,4$ & 0,92 & 0,96 & 0,89 \\
& $1,5,5$ & 0,93 & 0,95 & 0,89 \\
\hline
\end{tabular}

${ }^{1} \mathrm{RCP}$ - regresión por componentes principales, $\mathrm{CMP}$ - regresión por cuadrados mínimos parciales, CMPM - regresión por cuadrados mínimos parciales modificados.

${ }^{2}$ Orden de la derivada, longitud del segmento (nanómetros), longitud del segmento (nanómetros) de la primera suavización.

La información estadística de la calibración y de la validación cruzada para las ecuaciones generadas por CMPM y tratamiento matemático $1,5,5$ son presentadas en el Cuadro 3. Altos coeficientes de determinación $\left(\mathrm{R}^{2}>0,91\right)$, que son los valores de la curva, y bajos Errores Estándar de Calibración y Validación Cruzada (EEC, EEVC < 1,25) fueron obtenidos para
Cuadro 3. Variables estadísticas de la calibración y validación cruzada de la regresión por CMPM. Se usó el tratamiento matemático $1,5,5$ para el espectro NIRS de la composición nutricional de pastos. Universidad Zamorano, Honduras. 2005.

\begin{tabular}{llccc}
\hline \multicolumn{1}{c}{ Componente } & $\mathbf{N}^{1}$ & $\mathbf{R}^{\mathbf{2}}$ & EEC & EEVC \\
\hline PC, \% de MS & 119 & 0,96 & 0,43 & 0,60 \\
FND, \% de MS & 126 & 0,95 & 0,98 & 1,25 \\
FAD, \% de MS & 124 & 0,91 & 1,08 & 1,22 \\
\hline
\end{tabular}

${ }^{1} \mathrm{~N}$ - Número de muestras usadas para la calibración, $\mathrm{R}^{2}$ - Coeficiente de determinación.

EEC - Error estándar de calibración; EEVC - Error estándar de validación cruzada, MS - Materia seca.

las ecuaciones de PC, FND y FAD de acuerdo con estudios previos (Norris et al. 1976, Brooks et al. 1984, García-Ciudad et al. 1993, Woolnough y Foley 2002, Vasquez et al. 2004).

\section{NIRS versus los métodos de referencia}

Las ecuaciones NIRS predijeron con exactitud el contenido de PC, FND y FAD medidos por los métodos químicos de referencia de las 24 muestras utilizadas para la validación externa que no fueron utilizadas durante la calibración (Cuadro 4). Igualmente, las ecuaciones mostraron alta precisión $(\% \mathrm{CV}<5)$.

Cuadro 4. Estadística de la validación externa de las ecuaciones NIRS para el análisis de componentes nutricionales de pastos tropicales. Universidad Zamorano, Honduras. 2005.

\begin{tabular}{lccc}
\hline \multicolumn{1}{c}{ Componente } & $\mathbf{R}^{2 *}$ & EEV & \% CV \\
\hline PC, \% de MS & 0,98 & 0,25 & 4,5 \\
FND, \% de MS & 0,90 & 0,96 & 1,5 \\
FAD, \% de MS & 0,92 & 0,60 & 1,5 \\
\hline
\end{tabular}

* $\mathrm{R}^{2}$ - Coeficiente de determinación, EEC - Error estándar de validación; \%CV - Porcentaje del coeficiente de variación, MS - Materia seca. 


\section{CONCLUSIONES}

Este estudio estableció que PC, FND y FAD pueden ser medidas en forma exacta y precisa en un amplio rango de pastos tropicales cultivados en América Central por medio NIRS. Un mayor número de muestras de pastos tropicales cultivados en la región deben de ser colectados y analizados para incrementar el rango de acción, exactitud y precisión de las ecuaciones.

\section{LITERATURA CITADA}

AOAC. 2005. Official methods of analysis of AOAC International. 18 ed. Association of Official Analytical Chemists, Gaithersburg, MD. 1526 p.

Brooks, J; Anderson, M; Urness, PJ. 1984. Infrared reflectance analysis of forage quality for elk. Wildl. Manage. 48: 254.

Cozzolino, D. 2002. Uso de espectroscopía de reflectancia en infrarrojo cercano para análisis de alimentos animales. Agrociencia 6: 25-32.

Cozzolino, D; Fassio, A; Fernández, E. 2003. Uso de la espectroscopía de reflectancia en el infrarrojo cercano para analizar ensilaje de maíz. Agric. Tec. 63: 387-393.
FOSS NIRSYSTEMS. 2004. WINISI ${ }^{\circledR}$ manual del programa. Disponible en: http://www.winisi.com/

Garcia-Ciudad, A; Garcia-Criado, B; Perez-Corona, ME; Vazquez de Aldana, BR; Ruano, A. 1993. Application of near infrared reflectance spectroscopy to chemical analysis of heterogeneous and botanically complex grassland samples. Journal of Science of Food and Agriculture 63: 419-426.

Lascano, CE. 2002. Caracterización de pasturas para maximizar la producción animal. Archivos Latinoamericanos. Prod. Animal. 10(2): 126-132.

Norris, KH; Barnes, RF; Shenk, JS; Moore, JE. 1976. Predicting forage quality by NIR. Animal Science 43: 889-897.

Vasquez, D; Abadia, B; Arreaza, L. 2004. Uso de espectroscopía de reflectancia en el infrarrojo cercano (NIRS) para la caracterización nutricional del pasto Guinea y del grano de maíz. Disponible en: http:// www.engormix.com/aplicacion_espectroscopia_ reflectancia_infrarrojos_articulos_644_BAL.htm

Woolnough, AP; Foley, WJ. 2002. Rapid evaluation of pasture quality for a critically endangered mammal, the northern hairy-nosed wombat (Lasiorhinus krefftii). Wild. Res. 29: 91-100. 